\title{
Microprogramme en pédagogie de l'enseignement supérieur pour les enseignants : incidences sur la motivation et les stratégies d'apprentissage des étudiants
}

Mélanie Cabana and Denis Bédard

\section{CpenEdition}

\section{Journals}

Electronic version

URL: https://journals.openedition.org/ripes/3599

DOI: 10.4000/ripes.3599

ISSN: 2076-8427

\section{Publisher}

Association internationale de pédagogie universitaire

\section{Electronic reference}

Mélanie Cabana and Denis Bédard, "Microprogramme en pédagogie de l'enseignement supérieur pour les enseignants : incidences sur la motivation et les stratégies d'apprentissage des étudiants", Revue internationale de pédagogie de l'enseignement supérieur [Online], 37(3) | 2021, Online since 09 November 2021, connection on 27 September 2022. URL: http://journals.openedition.org/ripes/3599; DOI: https://doi.org/10.4000/ripes.3599

This text was automatically generated on 27 September 2022.

\section{c) (†)}

Creative Commons - Attribution-NonCommercial-ShareAlike 4.0 International - CC BY-NC-SA 4.0

https://creativecommons.org/licenses/by-nc-sa/4.0/ 


\title{
Microprogramme en pédagogie de l'enseignement supérieur pour les enseignants : incidences sur la motivation et les stratégies d'apprentissage des étudiants
}

\author{
Mélanie Cabana and Denis Bédard
}

\section{Introduction}

1 Aujourd'hui, il est possible de constater que plusieurs universités canadiennes proposent des programmes de formation aux enseignants du supérieur. Selon Potter et al. (2015), ces programmes de formation ont graduellement pris leur place en Amérique du Nord depuis les années 1970. Ils favorisent généralement le passage d'un enseignement transmissif à un enseignement centré sur l'apprentissage étudiant (Frenay et al., 2010; Taylor et Bédard, 2010). Dans la littérature, cette transformation de la pratique est généralement associée au paradigme de l'apprentissage (Angelo, 1997; Barr et Tagg, 1995).

2 Au Canada, les offres de formation sont élaborées selon diverses modalités. Certaines sont créditées, d'autres non. Certaines sont inscrites dans un parcours d'études de deuxième cycle, d'autres de troisième cycle et leur durée est variable. La durée de la formation serait un facteur déterminant si l'intention est d'amener des changements substantiels et durables dans les pratiques pédagogiques des enseignants qui, à leur tour, peuvent favoriser des apprentissages de qualité chez les étudiants (Ménard et al., 2017; Potter et al., 2015). Pour Potter et al. (2015), les «programmes de formation longs ", s'ils sont bien structurés et échelonnés sur une période d'au moins un an, sont susceptibles d'être bénéfiques. Cette durée est considérée comme favorable à l'apprentissage des connaissances et au développement des compétences en pédagogie 
de l'enseignement supérieur (Potter et al., 2015). Une telle formation devrait également favoriser un changement de croyances et d'attitudes face à l'apprentissage et à l'enseignement, contribuant ainsi au développement d'une identité professionnelle et d'une culture de l'enseignement centrées sur l'apprentissage étudiant (Bélisle, 2017).

3 Depuis 2009, l'Université de Sherbrooke (Canada) propose un programme volontaire de formation long: le MPES. Avec ses dix cours, le MPES propose aux enseignants des parcours de formation flexibles ${ }^{1}$, ponctués d'activités pédagogiques au choix permettant d'approfondir une diversité de thématiques en enseignement supérieur. La particularité du MPES est de proposer aux enseignants d'adopter une posture de praticien-chercheur (Bédard, 2014) dans l'esprit du Scholarship of Teaching and Learning (SoTL) (Bélisle et al., 2016). Les enseignants sont ainsi invités à réfléchir et à documenter tout changement qu'ils planifient apporter dans leur cours ou dans le programme auquel ils sont rattachés. En ce sens, les enseignants sont invités à aller audelà de l'acquisition de nouvelles connaissances (Bédard et Cabana, 2015).

4 Le MPES, dont l'ancrage théorique prend racine dans les approches sociocognitive et socioconstructiviste, envisage l'apprentissage dans une perspective de traitement de l'information et de coconstruction du savoir (Bédard et Cabana, 2015). Les activités pédagogiques offertes sont donc conçues de manière à engager activement l'étudiant dans les situations d'apprentissage et d'évaluation qui lui sont proposées, en lui proposant notamment de discuter, de débattre, de coopérer ou de collaborer. De plus, l'élaboration des activités s'inscrit dans une approche par compétences enchâssées dans une trajectoire de développement de compétences (Scallon, 2004; Tardif, 2006).

Cet article présente les résultats d'une étude qui a pour objectif de vérifier les incidences du MPES sur la motivation et les stratégies d'apprentissage des étudiants ayant suivi les cours d'enseignants ayant suivi la formation offerte au microprogramme. Pour mener à bien cette étude, nous avons adopté la perspective théorique dans laquelle est ancré le programme en question. L'étude s'appuie sur une enquête par questionnaire réalisée à l'hiver et l'automne 2015. Neuf enseignants ayant suivi la formation ont volontairement accepté que leurs étudiants répondent au questionnaire deux fois durant un semestre.

6 Après avoir présenté la recension des écrits et la problématique, le texte fait état des objectifs de l'étude, des cadres de référence et de nos hypothèses. Par la suite, la méthodologie utilisée est décrite, de même que les résultats de l'étude. Enfin, la discussion et la conclusion sont présentées.

\section{Problématique}

7 Selon Barr et Tagg (1995), le passage du paradigme de l'enseignement à celui de l'apprentissage invite les établissements d'enseignement supérieur à être au service de l'apprentissage. Selon ce paradigme, la finalité des établissements est de créer des environnements et des expériences d'apprentissage qui amènent les étudiants à découvrir et à construire leurs connaissances, comme leurs compétences. De surcroit, ces environnements devraient valoriser la coopération, la collaboration et le soutien entre les pairs apprenants (Barr et Tagg, 1995).

Depuis la parution du texte de Barr et Tagg en 1995, force est de constater que les enseignants sont de plus en plus nombreux à introduire les pédagogies actives à leurs 
pratiques. Ces transformations concernent des enseignants qui, partant d'un intérêt personnel, expérimentent de nouvelles approches pédagogiques dans leur salle de classe (Béchard, 2001) ou bien elles s'inscrivent dans une approche programme et touchent l'ensemble du parcours de formation (Bédard, 2019; Bélisle, 2011). Conséquemment, il est désormais possible d'accéder à une littérature abondante et diversifiée au sujet des pédagogies actives, ainsi qu'à leurs effets sur la motivation des étudiants et leurs stratégies d'apprentissage.

\subsection{Motivation des étudiants}

Comme la motivation représente une condition sine qua non à l'engagement et à la persévérance dans les études, plusieurs chercheurs se sont penchés sur ses caractéristiques. Selon Viau (2009a), le défi auquel font face les enseignants à l'université consiste davantage à maintenir la motivation des étudiants tout au long de leur parcours de formation, plutôt que tenter de la susciter ou de l'augmenter. De fait, des études réalisées au Canada et en Belgique (Bédard et Viau, 2001; Schmitz et al., 2010; Viau et al., 2004) montrent que les étudiants entrent généralement à l'université avec une motivation élevée. Leur persévérance dépend, elle, des interactions avec leur environnement et la manière dont ils perçoivent notamment les cours et leur sentiment d'efficacité. Pour Béchard et Bédard (2009), la correspondance entre les apprentissages réalisés et la réalité du milieu professionnel apparaissent comme un facteur important qui joue sur la perception des étudiants à l'égard du programme de formation. Selon ces auteurs, les étudiants estiment que les activités pédagogiques devraient contribuer au développement des compétences professionnelles attendues dans le milieu de pratique.

Plusieurs recherches (Bédard et Viau, 2001; Clerc et Brasselet, 2017; Dolman et Schmidt, 2006; Kozanitis, 2010; Lumpkin et al., 2015; Viau et al., 2004) indiquent que les pédagogies actives sont plus efficaces pour susciter la motivation des étudiants comparées aux pédagogies plus traditionnelles comme l'exposé magistral et les séminaires. Ces études montrent entre autres que les étudiants perçoivent les pédagogies actives plus engageantes et plus utiles à l'apprentissage. Dans les années 2000, Viau et ses collaborateurs (Bédard et Viau, 2001; Viau, Joly et Bédard, 2004; Viau et al., 2004) ont réalisé deux recherches au sujet des effets des pédagogies sur la motivation d'étudiants. Ces travaux menés dans deux universités québécoises francophones ont permis de sonder 7170 étudiants sur la façon dont ils percevaient les manières d'enseigner et de faire apprendre de leur enseignant. Les résultats de ces études révèlent que l'approche par projets est la pédagogie qui a motivé le plus les étudiants. Les pédagogies telles que les études de cas, l'apprentissage par problèmes (APP) et les ateliers ont, elles aussi, été identifiées comme étant motivantes.

11 D'autres recherches montrent que certaines pédagogies actives augmentent la motivation intrinsèque des étudiants (Clerc et Brasselet, 2017; Fukuzawa et al., 2017). Pour Ryan et Deci (2000), la motivation intrinsèque d'une personne réside dans trois besoins fondamentaux : le besoin d'autonomie, de compétence et d'affiliation sociale. Selon ces auteurs, plus une personne a le sentiment de pouvoir choisir, plus elle est intrinsèquement motivée. À titre d'exemple, Fukuzawa et al. (2017) ont examiné les effets de l'APP sur la motivation intrinsèque de 49 étudiants issus de deux spécialités (médecine légale ou anthropologie) et inscrits dans un cours de troisième année de 
premier cycle sur l'ostéologie humaine à l'Université de Toronto à Mississauga. Les résultats de cette étude montrent pour la plupart des étudiants une augmentation des trois déterminants de la motivation entre le début et la fin du cours. Les étudiants reconnaissaient que les apprentissages réalisés étaient applicables à d'autres disciplines et à des projets. À l'instar de Viau et de ses collègues, l'étude de Fukuzawa et al. (2017) montre également que la motivation est passée de très élevée au début du cours à très faible à la fin du cours, en particulier chez les étudiants ayant une plus grande connaissance de la matière.

12 Avec ces pédagogies, les étudiants sont invités à devenir des apprenants actifs. Mais face à ces nouveaux rôles et nouvelles responsabilités, il arrive que certains se montrent résistants (Bishop, Caston et King, 2014; Seidel et Tanner, 2013; Stover et Holland, 2018; Yadav et al., 2011). La recherche montre ainsi que les pédagogies actives peuvent susciter la motivation des étudiants, mais que cet effet ne peut être tenu pour acquis (Bishop et al., 2014; Stover et Holland, 2018; Yadav et al., 2011). Selon Viau (2009b), la qualité des apprentissages d'un étudiant dépend en grande partie de sa motivation, mais elle dépend aussi de sa capacité à mobiliser des stratégies d'apprentissage efficaces.

\subsection{Stratégies d'apprentissage}

Les théories cognitivistes postulent qu'apprendre est essentiellement une activité de traitement de l'information qui se réalise à différents niveaux: affectif, cognitif et métacognitif (Tardif, 1997). Pour Fayol (2011), il s'agit d'un processus mental complexe, dont le support est la mémoire. Ce processus suppose que l'étudiant encode de nouvelles informations, stocke ces informations dans la mémoire à long terme, puis les récupère lorsque nécessaire.

14 En éducation, des recherches montrent que les approches pédagogiques basées sur la résolution de problèmes et la collaboration entrainent une amélioration à long terme de la capacité à se remémorer les connaissances et elles permettent d'atteindre des niveaux de compétence plus élevés (Dolman et Schmidt, 2006; Galand et al., 2008). Dans ces études, il apparait que les pédagogies telles que l'apprentissage par projets et par problèmes, de même que l'apprentissage collaboratif favorisent un traitement efficace de l'information et améliorent, par conséquent, le développement de compétences transférables telles que les capacités réflexives, la résolution de problèmes et la communication.

L'étude de Bédard et Viau (2001) a aussi examiné les perceptions des étudiants à l'égard de leurs stratégies d'apprentissage et leurs perceptions des pédagogies. Les résultats montrent que les étudiants utilisent une diversité de stratégies d'apprentissage, et ce, quelle que soit la pédagogie. Les chercheurs ont toutefois constaté que les stratégies de sélection de l'information, comme souligner ou noter les idées importantes, ainsi que les stratégies d'élaboration, comme établir des liens entre la matière et ce qu'ils connaissent déjà, sont celles qui ont été le plus fréquemment utilisées. Les étudiants ont par ailleurs indiqué faire usage de stratégies d'organisation leur permettant de structurer la matière de manière à lui donner du sens. Ces résultats suggèrent que les étudiants ont traité l'information afin de mieux la comprendre et, comme dans les recherches de Frenay et Bédard (2011), cela apparait plus marqué lorsque les situations d'apprentissage sont ancrées dans la réalité du milieu professionnel. Les pédagogies 
plus traditionnelles, telles que l'exposé magistral et les séminaires de lecture, ont également favorisé l'utilisation de stratégies d'apprentissage, mais celles-ci sont apparues moins diversifiées et moins fréquentes.

16 Les recherches montrent que la discipline ou le domaine d'études influence aussi la manière d'apprendre des étudiants (Donald, 2002; Dumford et al., 2016). Donald (2002) a, par exemple, analysé plusieurs disciplines, notamment sous l'angle du développement intellectuel des étudiants, telles que l'analyse, la synthèse, l'évaluation critique et la création. Ses travaux mettent en évidence que certaines disciplines convergent vers des approches pédagogiques et montrent la nécessité de privilégier des stratégies d'apprentissage qui s'alignent sur ces mêmes pédagogies.

Notre recension des écrits montre l'importance des programmes à sensibiliser et à former les enseignants, de même qu'à développer des compétences pédagogiques. Mais que savons-nous de leur efficacité ou de leurs incidences? Les études qui se sont intéressées à cette question sont peu nombreuses (Ménard et al., 2017). Au Canada, en Angleterre, en Finlande et en Belgique, des études (Hanbury et al., 2008; Postareff et al., 2007, 2008; Potter et al., 2015; Stes et Van Petegem, 2011) établissent un lien positif entre une formation longue (au moins une année) et un changement de posture centrée sur l'apprentissage étudiant. Celles qui se sont intéressées plus spécifiquement à leurs retombées sur l'apprentissage des étudiants sont plus rares et ne montrent aucun résultat significatif (Cosnefroy et Fenouillet, 2008).

Depuis 2009, les enseignants de l'Université de Sherbrooke sont de plus en plus nombreux à s'inscrire au MPES, au cours duquel ils sont invités à se plonger au cœur d'une démarche réflexive centrée sur l'apprentissage étudiant (Bédard et Cabana, 2015). La présente étude a pour objectif de vérifier les incidences de cette formation sur la motivation et les stratégies d'apprentissage des étudiants d'enseignants qui ont été formés.

\section{Cadres de référence}

19 Nous avons adopté la perspective théorique dans laquelle est ancré le MPES, soit les approches sociocognitive et socioconstructiviste. La littérature montre que les pédagogies qui favorisent l'activation des connaissances antérieures, les conflits cognitifs, l'utilisation de stratégies d'apprentissage efficaces et le développement des capacités réflexives conduisent à la construction de nouvelles connaissances et au développement de compétences transférables (Dolman et Schmidt, 2006). Selon Galand et al. (2008), plus les étudiants perçoivent que les pédagogies facilitent le transfert des apprentissages, par exemple les pratiques pédagogiques contextualisées, plus ils disent utiliser des stratégies d'apprentissage efficaces, plus ils estiment que leurs pairs sont des ressources pour la construction de leurs connaissances et plus ils sont satisfaits de leur formation. De plus, ces chercheurs ont constaté que la résolution de problèmes concrets et authentiques aide les étudiants à réguler leurs apprentissages et à leur donner du sens. Pour Viau (2009b), un étudiant est actif lorsqu'il recourt de manière intentionnelle à des stratégies d'apprentissage qui sont, elles, fondées sur ses connaissances métacognitives et sur sa motivation. Il est donc attendu qu'un étudiant motivé s'engage et persévère davantage dans une activité qu'un étudiant qui ne l'est pas (Bédard, 2020; Kirschner et Hendrick, 2020). Lorsque l'engagement cognitif est à 
son plus haut niveau, l'étudiant mobilise des stratégies d'apprentissage qui lui conviennent et qui répondent aux exigences de l'activité.

Dans cette section, nous détaillons les trois concepts-clés au cœur de notre étude.

\subsection{Pédagogies de l'enseignement supérieur}

21 Dans le cadre du MPES, les participants sont introduits à une diversité de pédagogies actives. Quatre d'entre elles, davantage susceptibles d'être utilisées par les enseignants participant à notre étude, sont présentées :

22 - Apprentissage par problèmes (APP) : il est basé sur la participation active des étudiants qui réalisent une démarche d'analyse et de résolution de problèmes à partir de leurs connaissances antérieures et de leurs expériences vécues. La démarche est réalisée en petit groupe de cinq à quinze personnes (Bédard et Bourget, 2016).

23 - Approche par projets : elle est organisée autour de tâches complexes et guidée par une question ou un problème (Newton et Racey, 2015). Cette approche requiert des étudiants qu'ils adoptent de nombreuses stratégies d'apprentissage afin de mener à bien un projet, seul ou en équipe, qui comporte les mêmes étapes et les mêmes contraintes de réalisation que dans la vie professionnelle. Un projet aboutit à la conception et à la production d'un artéfact pouvant être présenté à des tiers à des fins d'évaluation.

24 - Travail en groupe : il consiste à regrouper les étudiants en petit groupe, généralement composé de trois à huit personnes, qui ont pour but de réaliser une tâche précise. Le but du travail en groupe est de faire travailler des étudiants ensemble de manière à faire converger les efforts intellectuels de chacun pour acquérir des connaissances sur un sujet particulier (Stover et Holland, 2018).

25 - Présentation orale ou par affiche : elle consiste à exposer une information particulière ou le fruit d'un travail devant ses pairs. La présentation peut faire appel à un support visuel comme l'affiche ou à tout autre moyen technologique. Elle peut être réalisée seule ou être combinée à un travail de groupe.

\subsection{Motivation scolaire}

26 La motivation scolaire est un phénomène complexe (CAPRES, 2021; Eccles et Wigfield, 2002; Ryan et Deci, 2000; Viau, 2009b). À l'instar de Viau (2009b), nous avons choisi d'aborder ce phénomène comme une dynamique : «Un phénomène qui tire sa source dans des perceptions que l'élève a de lui-même et de son environnement, et qui a pour conséquence qu'il choisit de s'engager à accomplir l'activité pédagogique qu'on lui propose et de persévérer dans son accomplissement, et ce, dans le but d'apprendre ». (p. 12)

27 Cette définition s'inspire particulièrement des travaux d'Eccles et de ses collaborateurs (Eccles, 2005), de Schunk et Pajares (2002), ainsi que de ceux de Ryan et Deci (2000). Dans l'approche sociocognitive, il est établi que les principales sources de la dynamique motivationnelle d'une personne résident dans ses perceptions (Viau, 2009b). Ainsi, les sources de la motivation correspondent à trois perceptions spécifiques :

28 - Perception de la valeur d'une activité : le jugement qu'un étudiant porte sur l'intérêt et l'utilité de l'activité pédagogique qui lui est proposée, en fonction des buts qu'il 
poursuit. L'intérêt se rapporte au plaisir intrinsèque et à toutes les émotions positives que suscite l'accomplissement d'une activité pédagogique (Ryan et Deci, 2000). L'utilité se rapporte plutôt aux bénéfices que l'étudiant retire de son accomplissement (Eccles et Wigfield, 2002).

- Perception de sa compétence: le jugement qu'un étudiant porte à l'égard de sa capacité à réussir une activité pédagogique particulière.

30 - Perception de contrôlabilité : le degré de contrôle qu'un étudiant a face au déroulement d'une activité ou d'une tâche qu'il réalise.

\subsection{Stratégies d'apprentissage}

31 Le modèle cognitif de l'apprentissage considère que les stratégies d'apprentissage facilitent l'acquisition, l'entreposage, le rappel et l'application de connaissances au moment de l'apprentissage (Boulet et al., 1996). Dans le cadre de notre étude, les stratégies cognitives et métacognitives sont prises en compte. Nous les présentons plus explicitement ci-après.

\subsubsection{Stratégies cognitives}

32 En milieu scolaire, les stratégies cognitives renvoient aux actions qu'un étudiant mobilise pour acquérir, intégrer et se rappeler les connaissances qui lui sont transmises (Viau, 2009b). En prenant appui sur les travaux de Boulet et al. (1996), de Frenay et Bédard (2011) et de Weinstein, Zimmerman et Palmer (1988), nous avons identifié les stratégies cognitives suivantes: de répétition, d'élaboration, d'organisation, de contextualisation et de sélection de l'information principale.

33 - Stratégies de mémorisation: répétition mentale, recopiage répétitif et révision répétitive (Boulet et al., 1996). Elles sont souvent utilisées lorsqu'un étudiant tente d'apprendre par cœur des informations. Selon Fayol (2011), elles permettent de maintenir les informations en mémoire de travail et ainsi d'éviter leur déclin avant qu'elles ne soient éventuellement transférées en mémoire à long terme. Néanmoins, l'auteur estime que ces stratégies ont une efficacité modeste lorsqu'elles sont utilisées seules.

34 - Stratégies d'élaboration: création de liens entre les nouvelles connaissances et les connaissances déjà acquises (Boulet et al., 1996). Elles peuvent aussi se traduire par des comportements tels que prendre des notes personnelles, paraphraser, reformuler la matière dans ses propres mots ou la résumer pour mieux l'apprendre et la comprendre, faire des analogies avec des situations familières, construire des métaphores ou réutiliser des travaux déjà réalisés et jugés pertinents.

35 - Stratégies d'organisation: mise en place cohérente, logique et structurée des informations présentées (Boulet et al., 1996). Elles peuvent se traduire par le regroupement, la classification ou catégorisation, la hiérarchisation ou la schématisation des informations.

36 - Stratégies de contextualisation : recherche de sens que l'étudiant effectue en apposant ou en adaptant l'information à différents contextes extérieurs à la classe (Bédard, 1999). Plus précisément, il s'agit de se représenter la matière dans des contextes professionnels variés, afin de se donner des repères plus signifiants (valeurs, attitudes et autres). 
- Stratégies de sélection de l'information principale: choix des idées clés de l'information à laquelle une personne, en situation de classe, est exposée (Weinstein et al., 1988). Reprenant les propos de Mayer (1988), Weinstein et al. (1988) expliquent qu'elles visent surtout à aider l'étudiant à focaliser son attention sur certaines informations, celles qu'il juge les plus importantes à l'accomplissement des buts qu'il poursuit.

\subsubsection{Stratégies métacognitives}

38 Les stratégies métacognitives font référence à la connaissance que l'étudiant a de ses propres stratégies cognitives et de la gestion qu'il exerce sur celles-ci. En nous inspirant des travaux de Boulet et al. (1996) ainsi que ceux de King et Kitchener (2002), nous avons identifié les stratégies métacognitives suivantes: d'autoévaluation, de régulation et des stratégies métacognitives (faisant référence à la pensée réflexive).

- Stratégies d'autoévaluation: évaluation du niveau de qualité et d'efficacité des stratégies cognitives mobilisées en situation d'apprentissage (Boulet et al., 1996). Ainsi, un étudiant qui mobilise des stratégies d'autoévaluation s'assure que son attention est entièrement consacrée à l'apprentissage, s'assure de bien comprendre la matière, prend conscience de ses progrès et anticipe les alternatives possibles de solutions.

40 - Stratégies de régulation : opérations que l'étudiant entreprend en vue d'apporter des correctifs à sa démarche d'apprentissage, et ce, en fonction des résultats obtenus lors de l'activité d'autoévaluation. Elles consistent donc à concentrer ses efforts sur ce qui n'est pas compris et modifier ses stratégies pour les rendre plus efficaces.

41 - Stratégies faisant référence à la pensée réflexive: différents niveaux de pensée analytique qu'un étudiant exerce sur les informations qui lui sont transmises (King et Kitchener, 2002).

\section{Hypothèses de recherche}

L'objectif de l'étude est de vérifier les incidences de la formation offerte à certains enseignants inscrits au MPES sur la motivation et les stratégies d'apprentissage de leurs étudiants. Pour ce faire, nous avons deux hypothèses principales :

Hypothèse 1: La formation reçue par l'enseignant influencera positivement la motivation des étudiants. S'en suivent deux sous-hypothèses (SH) :

- SH-1 : Cet effet sera significatif entre le début et la fin du cours;

45 - SH-2 : Cet effet sera significatif à la fois pour les exposés et les pédagogies actives.

46 Hypothèse 2: La formation reçue par l'enseignant influencera positivement la perception des étudiants qu'ils ont de l'utilisation des stratégies cognitives et métacognitives. S'en suivent trois sous-hypothèses $(\mathrm{SH})$ :

47 - SH-1 : Cet effet sera significatif entre le début et la fin du cours;

48 - SH-2 : Cet effet sera significatif à la fois pour les exposés et les pédagogies actives;

49 - SH-3 : Cet effet sera significatif pour chacune des disciplines/domaines. 


\section{Méthodologie}

50 Cette étude s'inscrit dans un programme de recherche longitudinale (2014-2017) s'intéressant aux pratiques d'enseignants en début de carrière provenant d'universités canadiennes et françaises (Ménard et al., 2017). Ce programme de recherche visait à comprendre et à documenter les retombées de la formation et de l'accompagnement pédagogiques que certains sollicitent et reçoivent. L'instrument de mesure (questionnaire) qui a servi à la collecte des données est commun aux deux études.

Figure . Une partie des données colligées et analysées est issue de l'étude de Ménard et al. (2017)

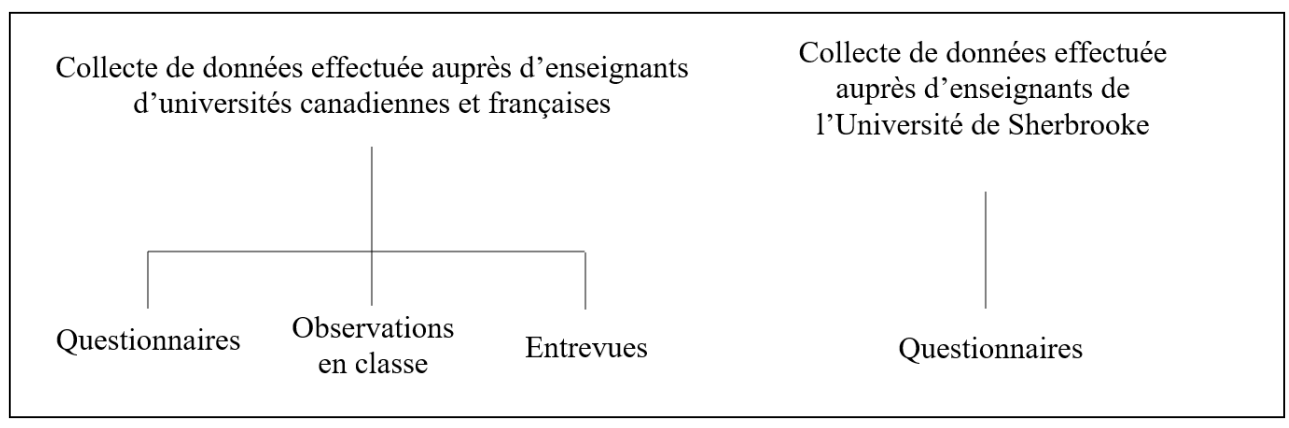

51 Il importe par ailleurs de préciser que la première auteure de cet article n'est d'aucune manière liée au MPES de l'Université de Sherbrooke, alors que le second auteur y enseigne certains cours. Dans cette section, nous présentons les participants, la procédure et les instruments de collectes de données, ainsi que le traitement et l'analyse des données recueillies.

\subsection{Participants}

Les enseignants qui ont participé à l'étude sont professeurs ou chargés de cours à l'Université de Sherbrooke. Ils ont suivi un ou trois cours du microprogramme. Au moment de recueillir les données, les participants avaient minimalement tous complété l'activité pédagogique obligatoire à l'entrée du MPES, soit Enseigner au supérieur. Leur participation à la présente étude a été volontaire. Deux vagues de recrutement réalisées lors d'une séance de cours offerte en ligne et par courriel ont permis de recruter neuf enseignants. Parmi ces enseignants, deux sont des femmes et sept des hommes. Comme il est possible de le voir dans le tableau 1, l'ancrage disciplinaire des participants est varié.

Tableau 1. Caractéristiques des enseignants

\begin{tabular}{|l|l|l|l|l|}
\hline & Sexe & Discipline & $\begin{array}{l}\text { Années } \\
\text { d'expérience }\end{array}$ & $\begin{array}{l}\text { Nombre de crédits complétés au } \\
\text { MPES }\end{array}$ \\
\hline 1 & F & Politique appliquée & 7 & 9 \\
\hline 2 & M & $\begin{array}{l}\text { Enseignement préscolaire et } \\
\text { primaire }\end{array}$ & 3 & 3 \\
\hline 3 & F & Enseignement collégial & 10 & 3 \\
\hline
\end{tabular}




\begin{tabular}{|l|l|l|l|l|}
\hline 4 & $\mathrm{M}$ & Chimie & 13 & 3 \\
\hline 5 & $\mathrm{M}$ & Chimie & 30 & 3 \\
\hline 6 & $\mathrm{M}$ & Chimie & 5 & 3 \\
\hline 7 & $\mathrm{M}$ & Chimie & 12 & 3 \\
\hline 8 & $\mathrm{M}$ & Informatique & 42 & 3 \\
\hline 9 & $\mathrm{M}$ & Ingénierie & 6 & 9 \\
\hline
\end{tabular}

Pour chacun de ces enseignants, un groupe-classe a été désigné pour participer à l'étude. Les groupes ciblés comptaient en moyenne 40 étudiants, allant de 15 à 63 . Au total, ce sont 360 étudiants qui ont été sollicités pour l'étude. La majorité des étudiants était inscrite au premier cycle d'études, alors que les autres étaient inscrits au deuxième cycle. Finalement, 252 étudiants ont répondu au questionnaire correspondant à un taux de réponse de $70 \%$. Le tableau 2 décrit les caractéristiques des groupes d'étudiants ayant rempli le questionnaire aux deux temps de la collecte (au début et à la fin du semestre).

Tableau 2. Caractéristiques des étudiants ayant répondu au questionnaire

\begin{tabular}{|c|c|c|c|c|c|c|c|c|c|c|}
\hline & \multirow{2}{*}{ Disciplines } & \multicolumn{2}{|c|}{$\operatorname{Sexe}^{a}$} & \multicolumn{6}{|c|}{ Année d'études } & \multirow{2}{*}{ Total } \\
\hline & & $M$ & $\mathrm{~F}$ & $1^{\text {re }}$ & $2^{\mathrm{e}}$ & $3^{\mathrm{e}}$ & $4^{\mathrm{e}}$ & $5^{\mathrm{e}}$ & $6^{\mathrm{e}}$ & \\
\hline 1 & Politique appliquée & 16 & 9 & 2 & 10 & 10 & 2 & 0 & 1 & 25 \\
\hline 2 & $\begin{array}{ll}\text { Enseignement } & \mathrm{au} \\
\text { préscolaire et } & \mathrm{au} \\
\text { primaire } & \end{array}$ & 3 & 21 & 24 & 0 & 0 & 0 & 0 & 0 & 24 \\
\hline 3 & $\begin{array}{l}\text { Enseignement } \quad \mathrm{au} \\
\text { collégial }^{\mathrm{b}}\end{array}$ & 5 & 12 & 13 & 4 & 0 & 0 & 0 & 0 & 17 \\
\hline 4 & Chimie & 9 & 8 & 0 & 11 & 4 & 1 & 1 & 0 & 17 \\
\hline 5 & Chimie & 18 & 18 & 1 & 32 & 5 & 0 & 0 & 0 & 38 \\
\hline 6 & Chimie & 5 & 11 & 0 & 0 & 17 & 0 & 0 & 0 & 17 \\
\hline 7 & Chimie & 4 & 5 & 3 & 0 & 1 & 5 & 0 & 0 & 9 \\
\hline 8 & Informatique & 33 & 3 & 26 & 12 & 1 & 0 & 0 & 0 & 39 \\
\hline 9 & Ingénierie & 26 & 13 & 34 & 1 & 2 & 1 & 1 & 0 & 39 \\
\hline & Total & 132 & 114 & 107 & 77 & 40 & 23 & 4 & 1 & 252 \\
\hline
\end{tabular}


${ }^{b}$ Au Canada, l'enseignement collégial est un ordre d'étude entre l'enseignement secondaire et l'enseignement universitaire. Il existe deux principaux cheminements : les formations préuniversitaires qui préparent pour l'université et les formations techniques qui peuvent mener directement à la pratique professionnelle ou à l'université.

\subsection{Collecte de données}

La collecte des données s'est déroulée aux semestres d'hiver et d'automne 2015. Un questionnaire papier a été administré par la première auteure en présence dans la salle de classe; une fois à la quatrième ou à la cinquième séance de cours (T1), puis une deuxième fois à la fin du semestre (T2). Dans le cadre de notre étude, nous avons utilisé le questionnaire élaboré par Ménard et al. (2017), disponible dans Cabana (2019), pour mesurer le niveau de motivation des étudiants, ainsi que la fréquence de leur utilisation des stratégies d'apprentissage, et ce, en tenant compte de deux situations pédagogiques distinctes: les exposés et les pédagogies actives. Les étudiants étaient invités à s'exprimer quant à leurs comportements en indiquant dans quelle mesure les énoncés du questionnaire leur correspondaient. Une échelle d'accord (Likert) à cinq niveaux a été utilisée pour évaluer leur niveau de motivation, puis une échelle de fréquence à quatre niveaux pour évaluer leur utilisation des stratégies d'apprentissage. Les étudiants étaient d'abord invités à évaluer leur niveau de motivation, de même que leurs stratégies d'apprentissage pendant les exposés, puis ils devaient choisir une pédagogie active parmi une liste à laquelle ils avaient déjà participé dans le cadre du cours et indiquer dans quelle mesure les énoncés du questionnaire leur correspondaient au moment de réaliser cette activité. Si aucune des pédagogies mentionnées n'avait été proposée par leur enseignant, les étudiants pouvaient en préciser une autre ou encore s'abstenir de compléter cette partie du questionnaire.

\subsection{Traitement et analyse des données}

Suivant la collecte des données, nous avons construit un score global (sur 5) des variables de la motivation et un score global (sur 4) des stratégies d'apprentissage. Les scores globaux ont été construits en calculant la moyenne des scores de l'ensemble des items pour chacune des variables. Dans le but de contrôler la qualité des données recueillies et analysées, une validation a été réalisée a posteriori sur ces scores. Ainsi, nous avons calculé l'indice alpha de Cronbach (IAC) et conservé les items présentant un coefficient supérieur à 0,70 (Osborne et Waters, 2002). Il importe de préciser que nous avons aussi souhaité explorer un maximum de variables et que, par conséquent, nous avons volontairement choisi de construire certains scores à partir de seulement deux items, alors qu'un minimum de trois est habituellement requis (tableau 3). Bien entendu, notre interprétation des résultats prend en compte cette limite.

Tableau 3. Statistiques de fiabilité des variables retenues

\begin{tabular}{|l|l|l|l|l|}
\hline \multirow{2}{*}{$\begin{array}{l}\text { Dimensions et sous-dimensions } \\
\text { (variables dépendantes) }\end{array}$} & \multicolumn{2}{|l|}{ Exposés } & \multicolumn{2}{l|}{ Pédagogies actives } \\
\cline { 2 - 5 } & Nb items & IAC & Nb items & IAC \\
\hline
\end{tabular}




\begin{tabular}{|l|l|l|l|l|}
\hline Motivation & 9 & 0,89 & 9 & 0,92 \\
\hline Perception de la valeur & 4 & 0,85 & 4 & 0,88 \\
\hline Perception de sa compétence & 3 & 0,89 & 3 & 0,88 \\
\hline Perception de contrôlabilité & 2 & 0,79 & 2 & 0,81 \\
\hline Stratégies d'apprentissage cognitives & & & & \\
\hline Stratégies de mémorisation & 2 & $*$ & 2 & 0,73 \\
\hline Stratégies d'élaboration & 3 & $*$ & 3 & $*$ \\
\hline Stratégies d'organisation & 3 & $*$ & 3 & 0,72 \\
\hline Stratégies de contextualisation & 3 & 0,87 & 3 & 0,92 \\
\hline Stratégies de sélection de l'information & 3 & $*$ & 3 & 0,70 \\
\hline Stratégies d'apprentissage métacognitives & & & & $*$ \\
\hline Stratégies d'autoévaluation & 2 & $*$ & 2 & $*, 90$ \\
\hline Stratégies de régulation & 6 & 0,87 & 7 & $*$ \\
\hline Stratégies faisant référence à la pensée réflexive & 4 & 2 & \\
\hline$*$ 0,70 & & & & \\
\hline
\end{tabular}

Pour répondre à la question de recherche et vérifier nos hypothèses, des analyses statistiques descriptives et inférentielles ont été réalisées sur les données présentant les perceptions des étudiants qui ont été recueillies. Premièrement, après les analyses statistiques descriptives, nous avons procédé à l'analyse descriptive des scores globaux qui ont été construits (moyennes et écart type). Ces analyses nous ont notamment permis de connaître la fréquence des pédagogies actives rapportées par les étudiants parmi la liste qui leur avait été fournie (exposé, APP, approche par projets, travail de groupe, présentation orale ou par affiche, et autre). Deuxièmement, nous avons effectué des analyses statistiques inférentielles pour mesures répétées. Un test d'hypothèse pour deux moyennes dépendantes ( $t$-test apparié ${ }^{2}$ a permis de comparer les mesures recueillies au début et à la fin du semestre. Considérant que l'échantillon de notre étude est suffisamment grand $(n>30)$, il nous a été possible de nous référer au théorème de la limite centrale pour la normalité de la distribution des moyennes d'échantillonnage (Field, 2013). Par ailleurs, comme notre échantillon d'enseignants a été constitué sur la base du volontariat, notre étude n'a pas pour ambition de généraliser les résultats. Néanmoins, les résultats de l'enquête permettent de décrire les retombées de la formation en pédagogie de l'enseignement supérieur sur l'apprentissage des étudiants. 


\section{Résultats}

57 Cette partie présente les résultats obtenus des analyses descriptives et inférentielles, de même que leur interprétation. Pour une meilleure compréhension des résultats, nous avons choisi de présenter en premier lieu la fréquence des situations pédagogiques sélectionnées par les étudiants. Aux points suivants, nous présentons les résultats obtenus à l'égard de l'évolution du niveau de motivation des étudiants et de leur utilisation des stratégies d'apprentissage dans un contexte de classe, et ce, en fonction des deux situations pédagogiques suivantes : les exposés et les pédagogies actives.

\subsection{Situations pédagogiques rapportées}

Tel qu'illustrée à la figure 2, une analyse descriptive a été effectuée sur les données perceptuelles recueillies pour connaître la fréquence des pédagogies actives rapportées par les étudiants.

Figure 2. Fréquence des pédagogies actives rapportées par les étudiants à la fin du semestre

Rappelons que les étudiants sollicités pour répondre au questionnaire ont été invités à évaluer leur motivation et leur perception de la fréquence d'utilisation des stratégies d'apprentissage en tenant compte de deux situations pédagogiques: l'exposé et les pédagogies actives. Ce faisant, tous les répondants ont évalué leurs perceptions à l'occasion d'un exposé, alors que leurs perceptions en situation de pédagogie active ont été distribuées en fonction du choix qu'ils ont fait parmi la liste des pédagogies actives qui leur ont été proposées.

\subsection{Motivation des étudiants}

Dans le cadre de cette étude, un test-t pour mesures répétées a été effectué sur les scores globaux de la variable motivation afin de vérifier l'hypothèse 1 et les soushypothèses (Hypothèse $1:$ la formation reçue par l'enseignant influencera positivement la motivation des étudiants. Sous-hypothèses : 1) cet effet sera significatif entre le début et la fin du cours; 2) cet effet sera significatif à la fois pour les exposés et les pédagogies actives). Les résultats obtenus ne montrent aucune différence significative du niveau de motivation des étudiants entre le début $(3,72 / 5)$ et la fin du cours $(3,73 / 5)$ ( $\mathrm{t}(251)=-0,18, \mathrm{p}<, 86)$ lorsque ceux-ci assistent aux exposés de l'enseignant. De la même façon, il n'y a aucune différence significative de la motivation entre le début $(3,96 / 5)$ et la fin du cours $(4,04 / 5)(t(165)=-1,60, p<, 11)$ lorsque l'enseignant leur propose une pédagogie active. Le tableau 4 présente ces résultats.

Tableau 4. Motivation des étudiants au début et à la fin du cours

\begin{tabular}{|l|l|l|l|l|l|l|l|}
\hline & \multicolumn{2}{|l|}{ Début du cours } & \multicolumn{2}{|l|}{ Fin du cours } & $\mathbf{t}$ & $\mathbf{d l}$ & $\mathbf{p}<$ \\
\cline { 2 - 8 } & moyenne & é.-t. & moyenne & é.-t. & & & \\
\hline $\begin{array}{l}\text { Motivation pendant } \\
\text { les exposés }\end{array}$ & 3,72 & 0,64 & 3,73 & 0,70 & $-0,18$ & 251 & 0,86 \\
\hline
\end{tabular}




\begin{tabular}{|l|l|l|l|l|l|l|l|}
\hline Motivation pendant une pédagogie active & 3,96 & 0,66 & 4,04 & 0,73 & $-1,60$ & 165 & 0,11 \\
\hline
\end{tabular}

$61 \mathrm{Au}$ cours de l'analyse des données, nous avons cependant constaté une différence significative de la motivation des étudiants inscrits dans les programmes en enseignement préscolaire et primaire, ainsi que collégial. Traités de manière isolée, ces résultats montrent une augmentation significative entre le début et la fin du cours, à la fois en situation d'exposés et de pédagogies actives. L'analyse des sources de la motivation permet de constater que cette augmentation était plus élevée à l'égard de leurs perceptions de la valeur accordée à la situation pédagogique $(\mathrm{t}(40)=-4,55, \mathrm{p}<$, 000 ) et de leurs perceptions de compétence $(t(40)=-4,86, p<, 000)$ (Viau, 2009b). Ces résultats sous-entendent que les enseignants des programmes en enseignement formés à la pédagogie ont proposé des exposés et des activités ayant une grande importance aux yeux des étudiants et représentant un défi qu'ils étaient en mesure de relever.

\subsection{Stratégies d'apprentissage}

Un test-t pour mesures répétées a également été effectué sur les variables des stratégies cognitives et métacognitives afin de vérifier l'hypothèse 2 et les soushypothèses (Hypothèse 2 : la formation reçue par l'enseignant influencera positivement la perception des étudiants qu'ils ont de l'utilisation des stratégies cognitives et métacognitives. Sous-hypothèses : 1 ) cet effet sera significatif entre le début et la fin du cours; 2) cet effet sera significatif à la fois pour les exposés et les pédagogies actives; 3) cet effet sera significatif pour chacune des disciplines/domaines). Les résultats obtenus ne montrent aucune différence significative de la fréquence d'utilisation des stratégies d'apprentissage des étudiants entre le début et la fin du cours pendant les exposés de leur enseignant.

Tableau 5. Fréquence des stratégies d'apprentissage des étudiants au début et à la fin du cours en situation d'exposés

\begin{tabular}{|l|l|l|l|l|l|l|l|}
\hline & \multicolumn{2}{|l|}{ Début du cours } & \multicolumn{2}{|l|}{ Fin du cours } & & \multirow{2}{*}{$\mathbf{t}$} & \\
\cline { 2 - 8 } & M l & $\mathbf{p}<$ \\
\hline Contextualisation & 2,47 & 0,66 & 2,50 & 0,76 & $-0,78$ & 251 & 0,44 \\
\hline Régulation & 2,83 & 0,56 & 2,84 & 0,59 & $-0,44$ & 251 & 0,66 \\
\hline
\end{tabular}

Lorsque les enseignants leur ont proposé une pédagogie active, les résultats indiquent cependant une augmentation de la fréquence d'utilisation des stratégies de contextualisation $(t(167)=-2,89, p<, 004)$, de sélection de l'information $(t(167)=-2,75$, $\mathrm{p}<, 007)$ et de régulation $(\mathrm{t}(170)=-2,11, \mathrm{p}<, 04)$.

Tableau 6. Fréquence des stratégies d'apprentissage des étudiants au début et à la fin du cours en situation de pédagogies actives

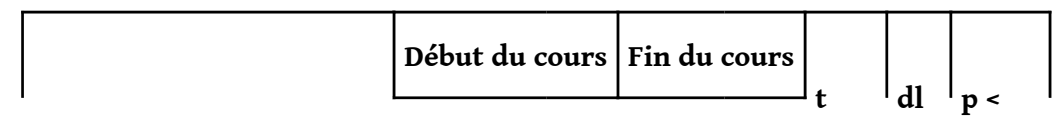




\begin{tabular}{|c|c|c|c|c|c|c|c|}
\hline & $M$ & É.T. & $M$ & É.T. & & & \\
\hline Mémorisation & 2,90 & 0,79 & 2,92 & 0,80 & $-0,37$ & 169 & 0,72 \\
\hline Organisation & 2,67 & 0,71 & 2,74 & 0,73 & $-1,31$ & 176 & 0,19 \\
\hline Contextualisation & 2,44 & 0,77 & 2,59 & 0,85 & $-2,89$ & 167 & $0,004^{*}$ \\
\hline Sélection de l'information & 3,03 & 0,57 & 3,14 & 0,68 & $-2,75$ & 167 & $0,007^{*}$ \\
\hline Régulation & 2,91 & 0,55 & 2,99 & 0,60 & $-2,11$ & 170 & $0,04^{* *}$ \\
\hline${ }^{*} \mathrm{p}<0,01{ }^{* *} \mathrm{p}<0,05$ & & & & & & & \\
\hline
\end{tabular}

Dans le contexte étudié, ces résultats suggèrent que les enseignants formés à la pédagogie ont favorisé le développement de stratégies d'apprentissage efficaces chez leurs étudiants en proposant des pédagogies actives. Frenay et Bédard (2011) soulignent que les stratégies de contextualisation, comme se représenter des contextes professionnels où les connaissances sont susceptibles d'être réinvesties et se représenter toute la culture du milieu professionnel, permettent aux étudiants de développer progressivement les compétences, habiletés et attitudes nécessaires dans le milieu de pratique. De plus, les étudiants qui utilisent les stratégies de sélection de l'information dirigent leur attention sur les informations qu'ils jugent les plus importantes et évitent, par conséquent, de se perdre dans les détails. Enfin, les stratégies de régulation permettent, quant à elles, de concentrer leurs efforts sur ce qui n'est pas compris et modifier leur manière de faire pour être plus efficaces.

En examinant la perception que les étudiants ont de leur utilisation des stratégies d'apprentissage en fonction de leur discipline, nous observons que les étudiants des programmes en enseignement préscolaire et primaire, de même que collégial ont augmenté significativement la fréquence d'utilisation des stratégies d'organisation, de contextualisation, de sélection de l'information et de régulation entre le début et la fin du cours (tableau 7). Plus de la moitié des répondants (56,1\%) indiquaient «le travail de groupe » comme pédagogie active, $22 \%$ «l'APP », $14,6 \%$ «la présentation orale ou par affiche », 4,9\% «l'approche par projets » et $2,4 \%$ la catégorie «autre ». Il est intéressant de constater que ces derniers aient utilisé des stratégies d'apprentissage efficaces en sélectionnant l'information importante, en organisant cette information de manière à lui donner du sens, en concentrant leurs efforts sur ce qui n'était pas compris et en régulant leur démarche d'apprentissage (Svinicki et McKeachie, 2014; Viau, 2009b).

Tableau 7. Différence de moyennes de la fréquence des stratégies d'apprentissage des étudiants des programmes en enseignement

\begin{tabular}{|c|c|c|c|c|c|c|c|}
\hline & \multicolumn{2}{|c|}{ Début du cours } & \multicolumn{2}{|c|}{ Fin $d u$ cours } & & \multirow{2}{*}{ dl } & \multirow{2}{*}{$\mathrm{p}<$} \\
\hline & M. & É.T. & M. & É.T. & & & \\
\hline Stratégies pendant les exposés & & & & & & & \\
\hline
\end{tabular}




\begin{tabular}{|l|l|l|l|l|l|l|l|}
\hline Contextualisation & 2,92 & 0,37 & 3,15 & 0,53 & $-2,57$ & 40 & $0,01^{* *}$ \\
\hline Régulation & 2,77 & 0,42 & 2,83 & 0,48 & $-0,83$ & 40 & 0,41 \\
\hline Stratégies pendant une pédagogie active & & & & & & & \\
\hline Mémorisation & 2,78 & 0,58 & 2,95 & 0,76 & $-1,43$ & 40 & 0,16 \\
\hline Organisation & 2,71 & 0,62 & 2,95 & 0,66 & $-2,52$ & 40 & $0,02^{* * *}$ \\
\hline Contextualisation & 2,88 & 0,55 & 3,21 & 0,52 & $-3,47$ & 40 & $0,001^{*}$ \\
\hline Sélection de l'information & 3,07 & 0,50 & 3,26 & 0,51 & $-2,64$ & 40 & $0,01^{* *}$ \\
\hline Régulation & 2,72 & 0,56 & 3,04 & 0,50 & $-3,31$ & 40 & $0,002^{* *}$ \\
\hline$* \mathbf{p}<\mathbf{0 , 0 0 1} * * \mathbf{p}<\mathbf{0 1} * * * \mathbf{p}<\mathbf{0 , 0 5}$ &
\end{tabular}

Outre les étudiants inscrits dans les programmes en enseignement, nous observons chez les étudiants inscrits dans le programme de chimie une augmentation significative entre le début et la fin du cours de la fréquence d'utilisation des stratégies de sélection de l'information ( $t(57)=-3,355, p<, 001)$ pendant une pédagogie active (tableau 8). Plus de la moitié $(52,5 \%)$ ont indiqué « l'APP » comme pédagogie active, $25 \%$ ont indiqué la catégorie « autre », $13,8 \%$ « la présentation orale ou par affiche » et $8,8 \%$ « le travail de groupe ». Le dépouillement de la catégorie «autre » permet de constater que d'autres types de pédagogies actives ont été rapportées par les étudiants comme les exercices, la résolution de problèmes, le laboratoire et l'enseignement entre pairs.

Tableau 8. Différence de moyennes de la fréquence des stratégies d'apprentissage des étudiants des programmes en chimie en situation de pédagogies actives

\begin{tabular}{|c|c|c|c|c|c|c|c|}
\hline & \multicolumn{2}{|c|}{ Début du cours } & \multicolumn{2}{|c|}{ Fin du cours } & \multirow{2}{*}{$t$} & \multirow{2}{*}{ dl } & \multirow{2}{*}{$\mathbf{p}<$} \\
\hline & M & É.T. & M & É.T. & & & \\
\hline Mémorisation & 3,36 & 0,59 & 3,29 & 0,66 & 0,67 & 58 & 0,51 \\
\hline Organisation & 2,93 & 0,64 & 2,87 & 0,70 & 0,74 & 57 & 0,46 \\
\hline Contextualisation & 2,24 & 0,72 & 2,28 & 0,75 & $-0,52$ & 57 & 0,60 \\
\hline Sélection de l'information & 3,20 & 0,46 & 3,43 & 0,52 & $-3,36$ & 57 & $0,001^{*}$ \\
\hline Régulation & 2,97 & 0,58 & 3,02 & 0,54 & $-0,91$ & 58 & 0,37 \\
\hline
\end{tabular}




\section{Discussion}

67 La présente étude avait pour objectif de vérifier les incidences du MPES sur la motivation et les stratégies d'apprentissage des étudiants d'enseignants qui ont suivi cette formation. Suivant cet objectif, deux hypothèses ont été formulées au sujet des enseignants ayant suivi les activités de formation du MPES.

H1. En fonction des résultats colligés et des analyses effectuées, le t-test apparié sur les scores globaux ne montre pas de différence significative entre la motivation des étudiants au début du cours et celles des étudiants à la fin du cours. Ce résultat est présent autant pour l'exposé que pour les pédagogies actives (voir le tableau 4).

Il importe de noter le «niveau » de motivation des étudiants au début des cours : 3,72/5 (exposés) et 3,96 (pédagogies actives). Ainsi, en moyenne, les étudiants indiquent être " en accord" (niveau 4) avec les énoncés qui traitent de la motivation. Ce niveau est donc élevé à l'entrée des cours. À la fin du cours, les scores ne varient pas de façon significative, même s'ils sont à la hausse. Ainsi, considérant le niveau élevé au début du cours, nous pouvons donc dire que la motivation positive des étudiants a été maintenue jusqu'à la fin. La littérature permet de conclure qu'il s'agit d'un résultat favorable en soi. En effet, dans le cadre d'autres recherches, la motivation des étudiants apparaît décliner entre le début d'un cours et la fin, comme entre le début d'une formation et sa conclusion (Bédard et Viau, 2001; Fukuzawa et al., 2017; Schmitz et al., 2010; Viau et al., 2004). Il importe cependant de mieux comprendre pourquoi. D'autres recherches sur le sujet, en particulier si elles mobilisaient une méthodologie mixte quali-quanti, permettraient de mieux expliquer le phénomène. De plus, des entretiens auprès des enseignants pourraient également permettre de documenter les choix faits et les décisions prises à lumière de la formation reçue.

Cela permettrait également d'éclairer les résultats obtenus chez les étudiants d'enseignants des programmes en enseignement préscolaire et primaire, de même que collégial. De façon distinctive, que ce soit au regard des exposés ou des pédagogies actives, la motivation de ces étudiants augmente de façon statistiquement significative. Autrement dit, les exposés et les activités pédagogiques proposés avaient une grande importance aux yeux des étudiants et représentaient un défi qu'ils étaient en mesure de relever. Selon les étudiants sondés, les enseignants ont principalement proposé des pédagogies actives telles que le travail de groupe $(56,1 \%)$, l'APP $(22,0 \%)$ et la présentation orale ou par affiche (14,6\%). Des études (Bédard et Viau, 2001; Galand et al., 2008; Lumpkin et al., 2015; Viau et al., 2004; Viau, Prégent et Forest, 2004) ont montré que ces pédagogies sont généralement perçues utiles par les étudiants pour développer les compétences professionnelles attendues dans le milieu de pratique. D'autres indiquent que les discussions au sein des groupes augmentent l'intérêt des étudiants pour la matière (Dolman et Schmidt, 2006). Considérant les autres résultats de cette recherche et l'état de l'art, ce résultat est remarquable. À nouveau, il serait important de pousser plus loin le type d'enquête qui pourrait être menée afin d'expliquer ces résultats, et ce, autant auprès des étudiants que des enseignants.

71 H2. En fonction des résultats colligés et des analyses effectuées, le t-test pour mesures répétées, sur les scores globaux ne montrent pas de différence significative entre les stratégies que les étudiants perçoivent utiliser au début du cours et celles des étudiants à la fin du cours en situation d'exposés (voir le tableau 5). Par contre, l'analyse des résultats entre le début et la fin du cours montre une différence significative entre les 
stratégies que les étudiants perçoivent utiliser lorsque des pédagogies actives sont proposées en classe (voir le tableau 6).

72 La première sous-hypothèse (SH1) est partiellement validée considérant que plusieurs étudiants utilisaient plus fréquemment certaines stratégies dont les stratégies de contextualisation, de sélection de l'information principale et de régulation à la fin du cours. La deuxième sous-hypothèse $(\mathrm{SH} 2)$ est invalidée puisque la différence d'utilisation des stratégies d'apprentissage entre le début et la fin du cours est significative seulement en situation de pédagogies actives. Il semble que malgré la formation pédagogique, les enseignants ne sont pas parvenus à accroitre la fréquence d'utilisation des stratégies de contextualisation et de régulation pendant les exposés. Les résultats indiquent cependant que les enseignants formés au MPES ont favorisé le développement de stratégies d'apprentissage efficaces chez leurs étudiants en proposant des pédagogies actives. Pour plusieurs chercheurs (Dolman et Schmidt, 2006; Galand et al., 2008), les pédagogies basées sur la résolution de problèmes et la collaboration favorisent un traitement efficace de l'information. Conséquemment, elles entrainent une amélioration à long terme de la capacité à se remémorer les connaissances et elles permettent d'atteindre des niveaux de compétence plus élevés. Galand et al. (2008) ont constaté que la résolution de problèmes concrets et authentiques aide les étudiants à réguler leurs apprentissages et à leur donner du sens. Dans la collaboration, chacun prend en considération le point de vue des autres et cherche par tous les moyens à aller au-delà des différences et des contradictions, et ce, afin de parvenir à une réponse commune ou acceptable pour le groupe. Les stratégies de régulation du groupe, mais également des individus dans le groupe sont-elles favorisées par une dynamique du conflit sociocognitif qui serait présente dans ces interactions?

Quant à la troisième sous-hypothèse, elle est également invalidée. En effet, seuls les enseignants des programmes en enseignement préscolaire et primaire, ainsi que collégial ont augmenté significativement la fréquence perçue d'utilisation des stratégies d'organisation, de contextualisation, de sélection de l'information et de régulation chez leurs étudiants entre le début et la fin du cours. Dès lors, il semble que les enseignants de ces programmes ont non seulement rehaussé la motivation de leurs étudiants, mais qu'ils ont également favorisé l'utilisation de stratégies d'apprentissage efficaces en contextualisant leurs exposés et en proposant des pédagogies actives (Frenay et Bédard, 2011). Hormis les enseignants des programmes en enseignement, les enseignants des programmes de chimie ont quant à eux augmenté significativement la fréquence perçue d'utilisation des stratégies de sélection de l'information principale chez les étudiants entre le début et la fin du cours.

74 Ces résultats font écho aux propos de Viau (2009b) et Galand et al. (2008) qui soutiennent que plus les étudiants perçoivent que les pédagogies favorisent l'acquisition de connaissances et le développement de compétences professionnelles, plus ils disent utiliser des stratégies d'apprentissage efficaces. D'après Viau (2009b), posséder de bonnes méthodes de travail s'avère être une caractéristique des étudiants performants, et une performance élevée semble être une source de motivation pour persévérer. Il serait intéressant de vérifier si la motivation des étudiants est positivement et statistiquement corrélée à leur fréquence d'utilisation des stratégies d'apprentissage. 


\section{Conclusion}

75 Comme il a été évoqué dans l'introduction, la formation des enseignants est devenue un enjeu clé pour les établissements d'enseignement supérieur, voire pour les gouvernements. À titre d'exemple, en France, l'arrêté du 8 février 2018 fixe le cadre national de la formation des maitres de conférences (MESRI, 2018). Il est prévu que cette « formation soit organisée au sein des établissements ou groupes d'établissements d'enseignement supérieur » (p. 23). Cette prise en charge de l'accompagnement et de la formation en pédagogie de l'enseignement supérieur par les établissements est présente au Canada depuis plus de 30 ans (Taylor et Bédard, 2010). Il est légitime de poser la question de l'incidence de ces formations sur le développement des compétences pédagogiques des professeurs, qu'elles soient courtes (ateliers thématiques de quelques heures) ou longues (cours de 2 ou 3 crédits -60 ou 90 heures).

Certaines recherches ont tenté d'identifier les retombées de la participation de dispositifs de formation des enseignants sur leurs conceptions des processus d'enseignement et d'apprentissage, de même que sur leurs pratiques d'enseignement (Bailly et al., 2015; Stes et Van Petegem, 2011). « Elles établissent généralement un lien positif entre une formation de longue durée de plus de 45 heures et l'évolution des conceptions et des approches" (Ménard et al., 2017, p. 4). Mais qu'en est-il de l'incidence de ces formations sur les étudiants de ces enseignants? C'est la question que la présente recherche a posée.

Derrière une telle question se profile "l'enjeu de démontrabilité ». Bien que l'idée soit de plus en plus fréquemment évoquée en enseignement supérieur, il est extrêmement difficile d'inférer des relations de causes à effets entre 1) une formation à l'enseignement, 2) des interventions pédagogiques et 3) la motivation et les stratégies d'apprentissage des étudiants. Force est de reconnaître que ce «modèle causal » est beaucoup plus complexe qu'il n'y paraît. Le contexte multifactoriel qui caractérise l'espace qui sépare les formations à l'enseignement au supérieur et la qualité de l'expérience d'apprentissage des étudiants ne peut justifier que les chercheurs abandonnent l'objectif de démontrer l'incidence de ces formations. Aucune recherche isolée ne pourra rendre compte de l'ensemble des facteurs et variables en cause. Le cumul de plusieurs recherches menées séparément, de courtes durées, comme la présente étude et d'autres, citées précédemment, peuvent permettre de jeter les bases d'un modèle explicatif de la valeur ajoutée des formations sur les enseignants qui les suivent et, en retour, sur les étudiants de ces enseignants. Toutefois, il importe de proposer des programmes de recherche ambitieux, mobilisant plusieurs chercheurs et établissements, avec des approches méthodologiques mixtes permettant de révéler toutes les facettes des changements en cause et de leurs incidences, et ce, dans une perspective longitudinale (trois à cinq ans). Seulement ce type de recherche permettra de rendre compte de l'évolution dans le temps des changements, tant chez les enseignants que chez les étudiants, sans parler des autres acteurs de la formation et des mesures d'accompagnement que sont les conseillers ou ingénieurs pédagogiques ou les tuteurs responsables d'offrir ces formations. Changer les conceptions et les pratiques des uns et des autres prend du temps, mais le temps peut devenir un allier dans une perspective d'approche programme.

78 Tant les programmes de formation offerts aux enseignants du supérieur que ceux qui encadrent la formation des étudiants permettent de rehausser l'offre en terme de 
qualité. Les individus et les cours pris isolément ne représentent qu'un aperçu limité de l'expérience d'apprentissage de chacun. Il importe donc de viser à inclure plusieurs des cours des programmes concernés, et ce, autant ceux qui s'adressent aux enseignants que ceux qui s'adressent aux étudiants. L'offre de formation du MPES, à titre d'exemple, s'inscrit dans une telle approche programme. Afin de comprendre le parcours d'apprentissage vécu par les enseignants qui s'y inscrivent, il importe de le documenter. De la même façon, afin de comprendre l'évolution de la motivation et des stratégies d'apprentissage des étudiants de ces enseignants, il importe également de décrire le contexte de formation dans lequel les cours qu'ils suivent s'inscrivent. Sans cet éclairage situationnel, sans un suivi dans le temps de leurs parcours, il sera difficile de modéliser adéquatement l'expérience d'apprentissage de chacun et de démontrer l'incidence des uns sur les autres.

\section{BIBLIOGRAPHY}

Angelo, T.-A. (1997). The Campus as Learning Community: Seven Promising Shifts and Seven Powerful Levers. AAHE Bulletin, 49(9), 3-6.

Bailly, B., Demougeot-Lebel, J. et Lison, C. (2015). La formation d'enseignants universitaires nouvellement recrutés : quelles retombées? Revue internationale de pédagogie de l'enseignement supérieur, 31(3). https://journals.openedition.org/ripes/990

Barr, R.-B. et Tagg, J. (1995). From teaching to learning: A new paradigm for undergraduate education. Change: The Magazine of Higher Learning, 27(6), 12-26.

Béchard, J.-P. (2001). L'enseignement supérieur et les innovations pédagogiques : une recension des écrits. Revue des sciences de l'éducation, XXVII(2), 257-281. http://doi.org/10.7202/009933ar Béchard, J.-P. et Bédard, D. (2009). Comprendre le monde des étudiants. Dans D. Bédard et J.-P. Béchard (dir.), Innover dans l'enseignement supérieur (p. 61-76). Presses Universitaires de France. Bédard, D. (2020). Motivation, engagement et persévérance des étudiants : quelques incidences pédagogiques. Le Tableau RUQ, 9(4), 1-2. http://pedagogie.uquebec.ca/le-tableau/motivation-etengagement-des-etudiants-quelques-incidences-pedagogiques

Bédard, D. (2019). PBL in medical education: A case study at the Université de Sherbrooke. Dans M. Moallem, W. Hung et N. Dabbagh (dir.), The Wiley Handbook of Problem-Based Learning (p. 459-482). John Wiley \& Sons.

Bédard, D. (2014). Être enseignant ou devenir enseignant dans le supérieur : telle est la question... de posture! Dans G. Lameul et C. Loisy (dir.), La pédagogie universitaire à l'heure du numérique (p. 97-110). De Boeck.

Bédard, D. (1999). Vers une pédagogie rigoureuse et renouvelée : l'impact des technologies de l'information et de la communication. Dans P. Bordeleau, C. Depover et L.-O. Pochon (dir.), L'école de demain à l'heure des technologies de l'information et de la communication (p. 93-106). Institut de recherche et de documentation pédagogique. 
Bédard, D. et Bourget, A. (2016). Préparer et animer une séance d'apprentissage par problèmes. Dans T. Pellacia (dir.), Comment mieux enseigner la médecine et les sciences de la santé (p. 45-90). De Boeck.

Bédard, D. et Cabana, M. (2015). La formation à l'enseignement à l'université : l'expérience du Microprogramme de troisième cycle en pédagogie de l'enseignement supérieur. Revue d'étude des Cantons-de-l'Est, 44, 41-55.

Bédard, D. et Viau, R. (2001). Le profil d'apprentissage des étudiantes et des étudiants de l'Université de Sherbrooke : résultats de l'enquête menée au trimestre d'automne 2000. [manuscrit non publié]. Vicerectorat à l'enseignement, Université de Sherbrooke, Sherbrooke, Québec.

Bélisle, M. (2017, 4-6 octobre). Comment innover dans nos pratiques d'enseignement? [communication]. Congrès de la Société internationale francophone d'éducation médicale (SIFEM), Université Aix-Marseille, France.

Bélisle, M. (2011). Perceptions de diplômés universitaires quant aux effets d'un programme professionnalisant et innovant sur leur professionnalisation en contexte de formation initiale [thèse de doctorat, Université de Sherbrooke]. Savoirs UdeS. http://savoirs.usherbrooke.ca/handle/ $11143 / 960$

Bélisle, M., Lison, C. et Bédard, D. (2016). Accompagner le Scholarship of Teaching and Learning. Dans A. Daele et E. Sylvestre (dir.), Comment développer le conseil pédagogique dans l'enseignement supérieur? Cadres de référence, outils d'analyse et de développement (p. 75-90). De Boeck.

Bishop, C.-F., Caston, M.-I. et King, C.-A. (2014). Learner-centered environments: Creating effective strategies based on student attitudes and faculty reflection. Journal of the Scholarship of Teaching and Learning, 14(3), 46-63. http://doi.org/10.14434/josotl.v14i3.5065

Boulet, A., Savoie-Zajc, L. et Chevrier J. (1996). Les stratégies d'apprentissage à l'universitéPresses de l’Université du Québec.

Cabana, M. (2019). Étude sur la motivation et les stratégies d'apprentissage d'étudiants dont les enseignants sont formés à la pédagogie de l'enseignement supérieur [mémoire de maitrise, Université de Sherbrooke]. Savoirs UdeS. http://hdl.handle.net/11143/16016

CAPRES (2021). Persévérance en enseignement supérieur. Dans Dossier CAPRES. http://www.capres.ca/ dossiers/perseverance-motivation

Clerc, J. et Brasselet, C. (2017). Enseigner la psychologie et la recherche par l'apprentissage par problème : effets sur les besoins psychologiques des étudiants. Pratiques psychologiques, 23, 243-253. http://doi.org/10.1016/j.prps.2017.04.002

Cosnefroy, L. et Fenouillet, F. (2008). Motivation et apprentissages scolaires. Dans P. Carré et F. Fenouillet (dir.), Traité de psychologie de la motivation (127-146). Dunod.

Dolman, D.-H.-J.-M. et Schmidt, H.-G. (2006). What do we know about cognitive and motivational effects of small group tutorials in problem-based learning? Advances in Health Sciences Education, 11, 321-336. http://doi.org/10.1007/s10459-006-9012-8

Donald, J.-G. (2002). Learning to think. Disciplinary perspectives. San Francisco, CA : Jossey Bass.

Dumford, A.-D., Cogswell, C.-A.et Miller, A.-L. (2016). The who, what, and where of learning strategies. The Journal of Effective Teaching, 16(1), 72-88. http://scholarcommons.usf.edu/ ehe_facpub/273 
Eccles, J.-S. (2005). Subjective task value and the Eccles et al. model of achievement-related choices. Dans A.-J Eliot et C.-S. Dweck (dir.), Handbook of competence and motivation (p. 105-121). Guilford.

Eccles, J.-S. et Wigfield, A. (2002). Motivational beliefs, values, and goals. Annual Review of Psychology, 53(1), 109-132. http://doi.org/10.1146/annurev.psych.53.100901.135153

Fayol, M. (2011). Un esprit pour apprendre. Dans E. Bourgeois et G. Chapelle (dir.), Apprendre et faire apprendre (2 éd., p. 11-21). Presses Universelles de France. (Ouvrage original publié en 2006)

Field, A.-P. (2013). Discovering statistics using IBM SPSS Statistics: And Sex and Drugs and Rock " $n$ " Roll. ( $4^{\mathrm{e}}$ éd.). Sage. (Ouvrage original publié en 2000)

Frenay, M. et Bédard, D. (2011). Le transfert des apprentissages. Dans E. Bourgeois et G. Chapelle (dir.), Apprendre et faire apprendre ( $2^{\mathrm{e}}$ éd., p. 125-137). Presses Universelles de France (Ouvrage original publié en 2006)

Frenay, M., Saroyan, A., Taylor, L., Bédard, D., Clement, M., Rege Colet, N., ... Kolmos, A. (2010). Accompagner le développement pédagogique des professeurs universitaires à l'aide d'un cadre conceptuel original. Revue française de pédagogie, 3(172), 63-76. http://doi.org/10.4000/rfp.2253

Fukuzawa, S., Boyd, C. et Cahn, J. (2017). Student motivation in response to problem-based learning. Collected Essays on Learning and Teaching, 10, 175-188. http://doi.org/10.22329/ celt.v10i0.4748

Galand, B., Bourgeois, E., Frenay, M. et Bentein, K. (2008). Apprentissage par problème et apprentissage coopératif : vers une intégration fructueuse? Dans Y. Rouiller et K. Lehraus (dir.), Vers des apprentissages en coopération : rencontres et perspectives (p. 139-163). Peter Lang.

Hanbury, A., Prosser, M. et Rickinson, M. (2008). The differential impact of UK accredited teaching development programmes on academics' approaches to teaching. Studies in Higher Education, 33(4), 449-483. http://doi.org/10.1080/03075070802211844

King, P.-M. et Kitchener, K.-S. (2002). The reflective judgment model: Twenty years of research on epistemic cognition. Dans B.-K. Hofer et P.-R. Pintrich (dir.), Personal epistemology: The psychology of beliefs about knowledge and knowing (p. 37-61). Lawrence Erlbaum, Publisher.

Kirschner, P. A. et Hendrick, C. (2020). How learning happens: Seminal works in educational psychology and what they mean in practice. Routledge.

Kozanitis, A. (2010). L'influence d'innovations pédagogiques sur le profil motivationnel et le choix de stratégies d'apprentissage d'étudiantes et d'étudiants d'une faculté d'ingénierie. Revue internationale de pédagogie de l'enseignement supérieur, 26(1). http://ripes.revues.org/385

Lumpkin, A., Achen, R.-M. et Dodd, R.-K. (2015). Focusing teaching on students: Examining student perceptions of learning strategies. Quest, 67(4), 352-366. http://doi.org/ 10.1080/00336297.2015.1082143

Mayer, R.-E. (1988). Learning strategies: An overview. Dans C. E. Weinstein, E. T. Goetz et P. A. Alexander (dir.), Learning and study strategies: Issues in assessment, instruction, and evaluation (p. 1122). Academic Press.

Ménard, L., Bédard, D., Leduc, D. et Gravelle, F. (2017). La formation pédagogique des nouveaux professeurs d'université : ses effets à court terme. Formation et profession, 25(3). http://formationprofession.org/files/numeros/18/v25_n03_413.pdf 
Ménard, L., Hoffmann, C. et Lameul, G. (2017). Effets de la formation à l'enseignement sur les pratiques des nouveaux enseignants-chercheurs. Recherche et formation, 84. http://doi.org/ 10.4000/rechercheformation.2771

Ministère de l'enseignement supérieur, de la recherche et de l'innovation (MESRI) (2018). Arrêté du 8 février 2018 fixant le cadre national de la formation visant à l'approfondissement des compétences pédagogiques des maîtres de conférences stagiaires. Journal officiel de la république française, 54. https://www.legifrance.gouv.fr/download/pdf? $\mathrm{id}=n T r Q 2 f B P Z D e 9 U w i a w e l a P W W U g v Y v f J 3 G c i R E w k W t l 3 E=$

Newton, G. et Racey, M. (2015). Evidence-informed strategies for undergraduate nutrition education: A review. Applied Physiology Nutrition and Metabolism, 40(7). https:// www.researchgate.net/publication/273446810_EvidenceInformed_Strategies_for_Undergraduate_Nutrition_Education_A_Review

Osborne, J.-W. et Waters, E. (2002). Four assumptions of multiple regression that researchers should always test. Practical Assessment, Research \& Evaluation, 8(2). http://pareonline.net/ getvn.asp? $=8 \& \mathrm{n}=2$

Postareff, L., Lindblom-Ylänne, S. et Nevgi, A. (2007). The effect of pedagogical training on teaching in higher education. Teaching and Teacher Education, 23(5), 557-571. http://doi.org/ 10.1016/j.tate.2006.11.013

Postareff, L., Lindblom-Ylänne, S. et Nevgi, A. (2008). A follow-up study of the effect of pedagogical training on teaching in higher education. Higher Education, 56, 29-43. http://doi.org/ $10.1007 / \mathrm{s} 10734-007-9087-\mathrm{z}$

Potter, M.-K., Hustra, E., Ackerson, T. et Prada, L. (2015). The effects of long-term systematic educational development on the beliefs and attitudes of university teachers. Higher Education Quality Council of Ontario.

Ryan, R.-M. et Deci, E.-L. (2000). Intrinsic and extrinsic motivations: Classic definitions and new directions. Contemporary Educational Psychology, 25, 54-67. http://doi.org/10.1006/ceps.1999.1020

Scallon, G. (2004). Évaluation des apprentissages dans une approche par compétences. Montréal : ERPI.

Schmitz, J., Frenay, M., Neuville, S., Boudrenghien, G., Wertz, V., Noël, B. et Eccles, J. (2010). Étude de trois facteurs clés pour comprendre la persévérance à l'université. Revue française de pédagogie, 172. http://rfp.revues.org/2217

Schunk, D.-H. et Parajes, F. (2002). The development of academic self-efficacy. Dans A. Wigfield et J.-S. Eccles (dir.), Development of achievement motivation (p. 16-31). Academic Press inc.

Seidel, S.-B. et Tanner, K.-D. (2013). “What if students revolt?” - Considering student resistance: Origins, options, and opportunities for investigation. Life Sciences Education, 12, 586-595. http:// doi.org/10.1187/cbe-13-09-0190

Stes, A. et Van Petegem, P. (2011). La formation pédagogique des professeurs dans l'enseignement supérieur : Une étude d'impact. Recherche et formation, 67, 15-30. https://

journals.openedition.org/rechercheformation/1360

Stover, S. et Holland, C. (2018). Student resistance to collaborative learning. International Journal for the Scholarship of Teaching and Learning, 12(2). http://doi.org/10.20429/ijsotl.2018.120208

Svinicki, M. et McKeachie, W.-J. (2014). McKeachie's teaching tips: Strategies, research, and theory for college and university teachers (14 éd.). Cengage Learning. (Ouvrage original publié en 1953) 
Tardif, J. (2006). L'évaluation des compétences. Documenter le parcours de développement. Chenelière Éducation.

Tardif, J. (1997). Pour un enseignement stratégique. L'apport de la psychologie cognitive. Les Éditions Logiques.

Taylor, N. et Bédard, D. (2010). Faculty development in Canadian universities. Dans A. Saroyan et M. Frenay (dir.), Building teaching capacities in universities: A comprehensive international model (p. 23-42). Stylus.

Viau, R. (2014). Savoir motiver les étudiants. Dans L. Ménard et L. St-Pierre (dir.), Se former à la pédagogie de l'enseignement supérieur (p. 235-254). Chenelière éducation/AQPC.

Viau, R. (2009a). L'impact d'une innovation pédagogique : au-delà des connaissances et des compétences. Dans D. Bédard et J.-P. Béchard (dir.), Innover dans l'enseignement supérieur (p. 184-198). Presses Universitaires de France.

Viau, R. (2009b). La motivation à apprendre en milieu scolaire. Éditions du Renouveau Pédagogique inc. (ERPI).

Viau, R., Joly, J. et Bédard, D. (2004). La motivation des étudiants en formation des maîtres à l'égard d'activités pédagogiques novatrices. Revue des sciences de l'éducation, 30(1) 163-176. http:// doi.org/10.7202/011775ar

Viau, R., Prégent, R. et Forest, L. (2004). Les façons d'apprendre des étudiantes et des étudiants de baccalauréat à l'École Polytechnique de Montréal. Bureau d'appui pédagogique, Direction de l'enseignement et de la formation. École Polytechnique.

Weinstein, C.-E, Zimmerman, S.-A. et Palmer, D.-R. (1988). Assessing learning strategies: The design and development of the LASSI. Dans C.-E., Weinstein, E.-T., Goetz et P.-A. Alexander (dir.), Learning and study strategies. Issues in assessment, instruction, and evaluation (p. 25-40). Academic Press inc.

Yadav, A., Subedi, D., Lundeberg, M.-A. et Bunting, C.-F. (2011). Problem-based Learning: Influence on students' learning in an electrical engineering course. Journal of Engineering Education, 100(2), 253-280. http://doi.org/10.1002/j.2168-9830.2011.tb00013.x

\section{NOTES}

1. Ce programme de formation est offert selon un parcours de neuf ou quinze crédits. Dans les universités québécoises, le nombre de crédit est déterminé pour évaluer la charge de travail requise pour atteindre les cibles de formation. Un crédit représente environ 45 heures de travail. Les heures de travail peuvent prendre la forme d'activités réalisées en classe, en laboratoire, en atelier ou en stage, d'activités réalisées hors classe, de manière synchrone ou asynchrone. Chaque cours du MPES est de 3 crédits.

2. Le terme "apparié » signifie que des mesures répétées ont été réalisées auprès des mêmes sujets. 


\section{ABSTRACTS}

Since 2009, more and more teachers at the Université de Sherbrooke and from other insitutions have been asking for the training offered by the Microprogramme en pédagogie de l'enseignement supérieur, during which they are invited to immerse themselves in a reflective process centered on student learning. What are the impacts of this "long term" program? The purpose of this study is to verify the impact of this training program on the motivation and learning strategies of the students of teachers who received this training. Perceptual data collected from 252 students from diverse disciplinary backgrounds enrolled in six programs (enseignement préscolaire et primaire, enseignement collégial, politiques appliquées, chimie, informatique et génie) shows that teachers trained in higher education pedagogy offered lectures and activities that helped keep their students motivated throughout the semester. More specifically, the data reveal that teachers coming from the programs "enseignement préscolaire et primaire", as well as "enseignement au collegial" have also used active learning that supports the development of contextualization, information selection and monitoring strategies in their students.

Depuis 2009, les enseignants de l'Université de Sherbrooke et d'autres établissements sont de plus en plus nombreux à solliciter la formation offerte au Microprogramme de $3 \mathrm{e}$ cycle en pédagogie de l'enseignement supérieur (MPES). À travers cette formation, ils sont invités à se plonger au cœur d'une démarche réflexive centrée sur l'apprentissage étudiant. Quels sont les impacts de cette " formation longue »? Notre étude a pour objectif de mesurer les incidences de ce programme de formation sur la motivation et les stratégies d'apprentissage des étudiants ayant suivi les cours d'enseignants formés par le MPES. Les données perceptuelles recueillies auprès de 252 étudiants inscrits dans six programmes de formation différents (enseignement préscolaire et primaire, enseignement collégial, politiques appliquées, chimie, informatique et génie) montrent que les enseignants formés à la pédagogie de l'enseignement supérieur ont proposé des exposés et des activités favorisant le maintien de la motivation de leurs étudiants tout au long du semestre. Les données révèlent également que les enseignants ont aussi utilisé des pédagogies actives favorisant l'usage de stratégies de contextualisation, de sélection de l'information principale et de régulation chez leurs étudiants. De façon plus particulière, il ressort que les enseignants issus des programmes en enseignement préscolaire et primaire, de même qu'en enseignement collégial ont contribué à rehausser significativement la motivation de leurs étudiants. De plus, les enseignants de ces programmes ont favorisé une augmentation significative de la fréquence d'utilisation de stratégies d'apprentissage variées en contextualisant leurs exposés et en proposant des pédagogies actives.

\section{INDEX}

Mots-clés: formation, enseignement supérieur, pédagogie active, motivation, stratégies d'apprentissage

\section{AUTHORS}

\section{MÉLANIE CABANA}

Université de Sherbrooke, Québec, Canada, Melanie.Cabana@USherbrooke.ca 


\section{DENIS BÉDARD}

Université de Sherbrooke, Québec, Canada, Denis.Bedard@USherbrooke.ca 